\title{
Sure I'm Sure: Prefrontal Oscillations Support Metacognitive Monitoring of Decision Making
}

\author{
(ํ) Martijn E. Wokke, ${ }^{1,2,3,4,5}$ Axel Cleeremans, ${ }^{1,2,3}$ and K. Richard Ridderinkhof ${ }^{4,5}$ \\ ${ }^{1}$ Consciousness, Cognition, and Computation Group, ${ }^{2}$ Center for Research in Cognition and Neurosciences, and ${ }^{3}$ Neuroscience Institute, Université Libre de \\ Bruxelles, 1050 Bruxelles, Belgium, and ${ }^{4}$ Amsterdam Brain and Cognition and ${ }^{5}$ Brain and Cognition, Department of Psychology, University of Amsterdam, \\ 1018 Amsterdam, The Netherlands
}

Successful decision making critically involves metacognitive processes such as monitoring and control of our decision process. Metacognition enables agents to modify ongoing behavior adaptively and determine what to do next in situations in which external feedback is not (immediately) available. Despite the importance of metacognition for many aspects of life, little is known about how our metacognitive system operates or about what kind of information is used for metacognitive (second-order) judgments. In particular, it remains an open question whether metacognitive judgments are based on the same information as first-order decisions. Here, we investigated the relationship between metacognitive performance and first-order task performance by recording EEG signals while participants were asked to make a "diagnosis" after seeing a sample of fictitious patient data (a complex pattern of colored moving dots of different sizes). To assess metacognitive performance, participants provided an estimate about the quality of their diagnosis on each trial. Results demonstrate that the information that contributes to first-order decisions differs from the information that supports metacognitive judgments. Further, time-frequency analyses of EEG signals reveal that metacognitive performance is associated specifically with prefrontal theta-band activity. Together, our findings are consistent with a hierarchical model of metacognition and suggest a crucial role for prefrontal oscillations in metacognitive performance.

Key words: consciousness; decision making; EEG; metacognition

\section{Significance Statement}

Monitoring and control of our decision process (metacognition) is a crucial aspect of adaptive decision making. Crucially, metacognitive skills enable us to adjust ongoing behavior and determine future decision making when immediate feedback is not available. In the present study, we constructed a "diagnosis task" that allowed us to assess in what way first-order task performance and metacognition are related to each other. Results demonstrate that the contribution of sensory evidence (size, color, and motion direction) differs between first- and second-order decision making. Further, our results indicate that metacognitive performance specifically is orchestrated by means of prefrontal theta oscillations. Together, our findings suggest a hierarchical model of metacognition.

\section{Introduction}

Monitoring and control of our decision process (metacognition) (Flavel, 1979; Fleming and Dolan, 2012) is a crucial aspect of adaptive decision making. For instance, a doctor who is not very

Received April 21, 2016; revised Oct. 14, 2016; accepted Nov. 4, 2016.

Author contributions: M.E.W. and K.R.R. designed research; M.E.W. performed research; M.E.W. analyzed data; M.E.W., A.C., and K.R.R. wrote the paper.

This work was supported by Amsterdam Brain and Cognition (Talent Grant to M.E.W.), the European Research Council (Advanced Grant RADICAL to A.C.) and by the Belgian Science Policy Office (Interuniversity Poles of Attraction Grant P7/33 to A.C.). A.C. is a research director of the National Fund for Scientific Research. We thank Sarah L. Knot for help in collecting the data and Simon van Gaal and two anonymous reviewers for useful comments.

The authors declare no competing financial interests.

Correspondence should be addressed to Martijn E. Wokke, Université Libre de Bruxelles, Consciousness, Cogni-

tion and Computation Group, Department of Psychology, Avenue Franklin Roosevelt 50, 1050 Brussels, Belgium. E-mail:martijnwokke@gmail.com.

DOI:10.1523/JNEUROSCI.1612-16.2016

Copyright $\odot 2017$ the authors $\quad 0270-6474 / 17 / 370781-09 \$ 15.00 / 0$ confident about a diagnosis will prescribe additional tests; a tennis player who just executed a drop shot will assess the likelihood of the shot being successful to determine her next move. Crucially, such metacognitive skills enable us to adjust ongoing behavior and determine future decision making when immediate feedback is not available. Despite the obvious importance of metacognition, little is known about how our metacognitive system operates or how first-order performance and metacognition (second-order performance) are related to each other. In particular, it remains an open question whether first- and second-order judgments are based on the same information (Steinhauser and Yeung, 2010; Desender et al., 2016).

Metacognitive judgments are often viewed as being dependent on the very same processes that underpin first-order decisions. From this perspective, the information available for metacognitive computations is directly dependent on the quality 
and quantity of accumulation of sensory evidence (Kiani and Shadlen, 2009; Kiani et al., 2014). In contrast, dissociations between first- and second-order performance (Weiskrantz et al., 1974; Del Cul et al., 2009; Rounis et al., 2010; Harsay et al., 2012; Hebart et al., 2016; King and Dehaene, 2014; Fleming et al., 2015) suggest that metacognition and first-order task performance are supported by differential (though related) sources of information (Cleeremans et al., 2007; Yeung and Summerfield, 2012; Charles et al., 2014; Maniscalco and Lau, 2016). It has been proposed that dissociations between first- and second-order performance are the result of differences in availability of supporting information (Baranski and Petrusic, 1998; Del Cul et al., 2009; Yeung and Summerfield, 2012; Fleming et al., 2015). Further, hierarchical models of metacognition hold that sensory evidence used for first-order performance becomes susceptible to accrual of noise and signal decay when arriving at the stage at which this information is being used by the metacognitive system (Pleskac and Busemeyer, 2010; Maniscalco and Lau, 2016).

Over the last decade, first-order decision making has been strongly linked to trial-by-trial electrophysiological cortical oscillatory dynamics (Siegel et al., 2012). For instance, recent findings have associated theta-band activity with the rate of evidence accumulation and integration (van Vugt et al., 2012; WerkleBergner et al., 2014), whereas activity in the beta band has been shown to predict behavioral choices (Donner et al., 2007; Donner et al., 2009; Haegens et al., 2011) and seems to be important for maintenance of persistent activity (Engel and Fries, 2010; Siegel et al., 2012; Kloosterman et al., 2015). Despite mounting evidence of an intrinsic relationship between first-order decision making and neural oscillatory activity, it remains unknown how oscillatory dynamics relate to second-order decision making.

In the present study, we constructed a task in which participants were asked to make a "diagnosis" after seeing a sample of fictitious patient data (a pattern of colored moving dots of different sizes). The patterns provided probabilistic information about patient health according to contingencies unknown to the participant; participants were to learn these contingencies explicitly or implicitly (Cleeremans et al., 1998) and to diagnose each patient as accurately as possible. On each trial, participants rated both the quality and the reasoning strategy of their decision. Our task design allowed us to assess the relationship between fluctuations in electrophysiological oscillatory activity and changes in firstorder decision accuracy, metacognitive judgment adequacy, and strategy judgment. In addition, we were able to test how different sources of sensory evidence (size, color, and motion direction) contributed to first- and second-order task performance. To capture these behavioral and electrophysiological relationships, we applied the multiple regression method (Rousselet et al., 2009; Cohen and Cavanagh, 2011).

\section{Materials and Methods}

Participants. Thirty-eight participants (28 females, mean age $=23.1$, $\mathrm{SD}=6.55)$ took part in this study for financial compensation. To investigate how changes in task accuracy, metacognitive adequacy, and metacognitive strategy (Berry and Dienes, 1993; Price and Norman, 2008) related to neural oscillatory activity, we focused our analyses on those participants who exhibited variability in both their first- and secondorder decisions and metacognitive strategy. We therefore excluded participants who performed at chance level throughout the whole experiment, who almost exclusively indicated to have made decisions of very poor quality (resulting in $<50$ "high-quality decision" trials), and who almost exclusively guessed (resulting in $<50$ intuitive and rational trials; see below). Five participants were excluded because of failed EEG recordings. In these participants, we observed excessive noise in more

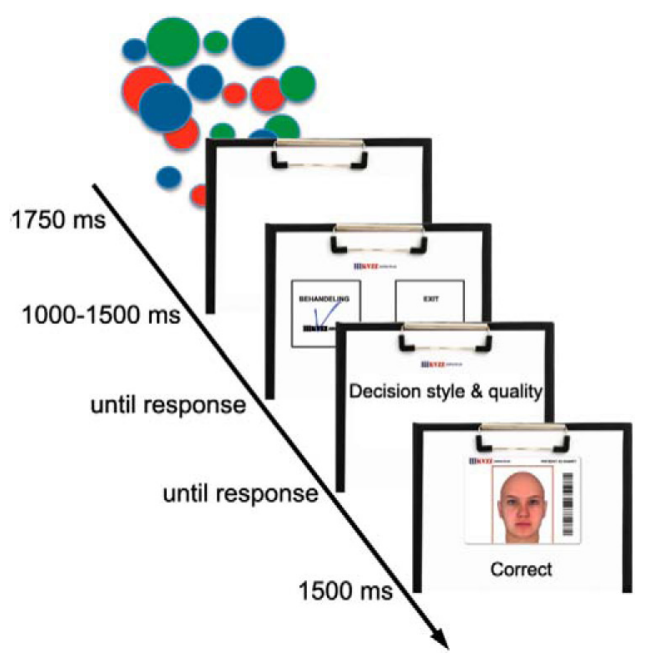

Figure 1. Task design. Participants made a "diagnosis" based on a pattern of moving dots that contained information indicative of illness. On each trial, participants provided judgments about the quality of their decisions and what kind of decision strategy that they used.

than half of all trials (partially due to our long epoch; see below). A total of 19 participants were included for further analyses. All participants had normal or corrected-to-normal vision and all were naive to the purpose of the experiment. All procedures complied with international laws and institutional guidelines and were approved by the Ethics Committee of the Psychology Department of the University of Amsterdam and all participants provided their written informed consent before the experiment.

Task design. Stimuli were presented full screen $(1024 \times 768$ pixels $)$ on a 17-inch Dell TFT monitor with a refresh rate of $60 \mathrm{~Hz}$. The monitor was placed at a distance of $\sim 90 \mathrm{~cm}$ in front of each participant so that $1 \mathrm{~cm}$ subtended a visual angle of $0.64^{\circ}$. On each trial, a sample of fictitious patient data was presented, which consisted of blue, red, and green circles of different sizes $(14,24$, and 34 pixels in diameter) that moved in three different directions $\left(45^{\circ}, 135^{\circ}\right.$, or $315^{\circ}$; Fig. 1) against a white background.

During the experiment, one color, one size, and one motion direction was indicative of illness (e.g., the color blue, medium size, and motion left upwards). The sample was positive if the presence of a combination of the indicative size, color, and motion direction exceeded a certain threshold (i.e., the criterion was set at $160 \%$, see below). The task parameters were based on data from an extensive pilot study and were set so as to measure trial-by-trial fluctuations in task accuracy, metacognitive adequacy, and metacognitive strategy. Metacognitive adequacy was based on decision quality ratings (see below). A high value was awarded when participants rated a correct decision to be a high-quality decision or when an error was rated as being of low quality and vice versa. On each trial, the percentage of each feature (size, color, and motion direction) was randomly set between $10 \%$ and $70 \%$ (steps of $10 \%$ ) until the total percentage of each separate feature added up to $100 \%$ (e.g., $30 \%$ small circles, $40 \%$ medium circles, and 30\% large circles). On "positive sample" trials, the percentages were randomly set until the sum of the percentages of the three indicators varied between $160 \%$ and $210 \%$. Importantly, participants had to pay attention to all three indicators to perform the task correctly. Paying attention to only one indicator was not sufficient. For instance, a high number of blue circles (e.g., 70\%) could belong to a negative sample depending on the percentage of the other two indicators. The task therefore required participants to discriminate conjunctively between the features.

The stimulus was presented for $\sim 1750 \mathrm{~ms}$, during which the circles were displaced 7 pixels per screen refresh in 1 of 3 possible directions. At any moment during stimulus presentation, a total of 600 circles were on the screen. Each trial started with a blank screen (jittered between 1000 and $1800 \mathrm{~ms}$ in steps of $100 \mathrm{~ms}$ ) on which the words "loading patient data" were centrally presented. After stimulus presentation $(\sim 1750 \mathrm{~ms})$, a blank screen (jittered between 1000 and $1500 \mathrm{~ms}$ with a $50 \mathrm{~ms}$ step) was 
presented to avoid the influence on prolonged evidence accumulation (Yeung and Summerfield, 2012; Hebart et al., 2016), followed by an image of a clipboard (Fig. 1) on which "sick" or "exit" had to be ticked by pressing a left or right button (indicating a positive or negative sample, respectively).

Next, participants had to rate how they had arrived at their diagnosis (strategy judgment). Participants could indicate whether their first-order decision was based on a pure guess (e.g., flipping a coin), was made intuitively (i.e., prereflective, described as the feeling of knowing what to decide without explicitly knowing why; Berry and Dienes, 1993; Price and Norman, 2008), or rationally (i.e., reflective, knowing what to decide based on explicit knowledge) by pressing the 1, 2, or 3 key, respectively. Participants then provided their estimate about the quality of their decision on a scale ranging from 1 to 5 (by pressing the $1-5$ keys). Participants were instructed to assign a low value to a diagnosis that they experienced to be of poor quality and a high value to a diagnosis that they considered to be of high quality. Participants were encouraged to make use of the whole scale. Finally, participants received feedback about their firstorder (diagnosis) decision (Fig. 1).

The experiment lasted $\sim 2.5 \mathrm{~h}$ and consisted of 512 trials divided into 8 blocks. After each block, the metacognitive scales (strategic judgment and judgment accuracy) were explained again to make sure that the meaning of the scales was understood properly throughout the entire experiment. Within each block, negative and positive samples were presented in pseudorandom order. Stimuli were presented using Presentation software (Neurobehavioral Systems).

Behavioral analyses. To determine whether first-order decision accuracy and metacognitive adequacy differed depending on strategic judgment, we calculated first-order task sensitivity $\left(d_{\mathrm{a}}\right.$, a variant of $d^{\prime}$ that takes unequal variance into account; see Macmillan and Creelman, 2004), metacognitive sensitivity ( meta- $d_{\mathrm{a}}$ ), and metacognitive efficiency (meta- $d_{\mathrm{a}}-d_{\mathrm{a}}$; Maniscalco and Lau, 2012; Fleming and Lau, 2014) for rational and intuitive decisions and guesses separately. First-order task sensitivity $\left(d_{\mathrm{a}}\right)$ and metacognitive sensitivity $\left(\right.$ meta- $\left.d_{\mathrm{a}}\right)$ are bias-free measures of the ability to detect a signal from noise (a sick sample in this experiment) and the ability to distinguish between good and bad decisions, respectively (both in units of first-order $d_{\mathrm{a}}$ ). By subtracting $d_{\mathrm{a}}$ from meta- $d_{\mathrm{a}}$ (metacognitive efficiency), we were able to determine metacognitive sensitivity relative to different levels of first-order task performance (Fleming and Lau, 2014). The latter is important because metacognitive sensitivity is known to be influenced by first-order task performance (Fleming and Lau, 2014). We performed multivariate repeated-measures analyses of variance (MANOVA) on first- and second-order task performance as dependent variables and strategic judgment (with three levels: rational, intuitive, and guess) as the independent variable.

To determine whether different stimulus parameters (size, color, and motion direction) contributed differentially to task accuracy and metacognitive adequacy, we performed robust multiple linear regressions, resulting in the following linear equations:

$$
\begin{aligned}
& \mathrm{Y}_{1}=\mathrm{INT}+\mathrm{b}_{1} \text { ColorEv }+\mathrm{b}_{2} \text { SizeEv }+\mathrm{b}_{3} \text { MotionEv }+\mathrm{b}_{4} \text { MetaAdeq }+\mathrm{E} \\
& \mathrm{Y}_{2}=\mathrm{INT}+\mathrm{b}_{1} \text { ColorEv }+\mathrm{b}_{2} \text { SizeEv }+\mathrm{b}_{3} \text { MotionEv }+\mathrm{b}_{4} \text { TaskAcc }+\mathrm{E}
\end{aligned}
$$

In this equation, $Y_{1}$ is the data vector containing first-order task performance scores, $Y_{2}$ is the data vector containing metacognitive adequacy scores, INT is the intercept, and $E$ is unexplained variance. The stimulus parameters ColorEv, SizeEv, and MotionEv ranged between 1 and 7, indicating the amount of evidence (percentage divided by 10) of each indicator (size, color, and motion direction, respectively) present on each trial. We rescored decision quality ratings (see above) such that they now reflected the adequacy of the metacognitive judgment (MetaAdeq). A high value was awarded when participants rated a correct decision to be a high-quality decision or when an error was rated as being of low quality; similarly, a low value was awarded when an error was rated as a highquality decision or when a correct was rated as a low-quality decision. This value of metacognitive adequacy could vary between 1 and 5 (i.e., 5 points were awarded when a correct decision received a 5-point rating or when an error received a 1-point rating; 4 points were awarded when a correct decision received a 4-point rating or when an error received 2 points, etc.). The values for task accuracy (TaskAcc) varied between 0 and 1 (incorrect and correct, respectively). To make the TaskAcc predictor less binary, we grouped all trials into 100 bins per participant. We adopted the multiple linear regression method to parcel out variance caused by different experimental settings (e.g., the varying amount of size, color, and motion direction evidence present in the stimulus on each trial) and behavioral variables (e.g., task accuracy or metacognitive adequacy). In this way, the unique contribution of each variable could be observed, controlling for shared variance among the different variables (Cohen and Cavanagh, 2011). To be able to compare $b$ values across participants and between behavioral variables (e.g., between task accuracy and metacognitive adequacy), we standardized $b$ values by scaling the coefficients by their SDs. All behavioral analyses were performed using MATLAB version 12.1 software (The MathWorks Inc.), type 2 SDT scripts (Maniscalco and Lau, 2012: http://www.columbia.edu/ bsm2105/type2sdt/), and SPSS version 22.0 (IBM).

EEG measurements and analyses. EEG was recorded and sampled at $1048 \mathrm{~Hz}$ using a Biosemi ActiveTwo 64-channel system, with four additional electrodes for horizontal and vertical eye movements, each referenced to their counterpart (Biosemi). High-pass filtering $(0.5 \mathrm{~Hz})$, additional low-pass filtering $(100 \mathrm{~Hz})$, and a notch filter $(50 \mathrm{HZ})$ were used. Next, we down-sampled to $512 \mathrm{~Hz}$, after which eye movements were corrected on the basis of independent component analysis (Vigário, 1997). The data were epoched -1 to $+4 \mathrm{~s}$ surrounding stimulus onset. We removed trials containing irregularities due to EMG or other artifacts by visually inspecting all trials. To increase spatial specificity and, to filter out deep sources, we converted the data to spline Laplacian signals (Cohen, 2014). Subsequently, per participant and per electrode, the average of all trials was subtracted from each individual trial to obtain the nonphase-locked power (Kalcher and Pfurtscheller, 1995; Donner and Siegel, 2011; Kloosterman et al., 2015). Next, we used a sliding window Fourier transform (Mitra and Pesaran, 1999) at window length $400 \mathrm{~ms}$ and step size $50 \mathrm{~ms}$ to calculate the time-frequency representations of the EEG power (spectrograms) for each channel and each trial. We used a single Hanning taper for the frequency range $2-30 \mathrm{~Hz}$ (frequency resolution: $2.5 \mathrm{~Hz}$, bin size: $1 \mathrm{~Hz}$; Kloosterman et al., 2015). Power modulations were characterized as the percentage of power change at a given time and frequency bin relative to baseline power value for that frequency bin. The baseline was calculated as the mean power across the prestimulus interval (from -0.3 to $0 \mathrm{~s}$ relative to stimulus onset). All signal processing steps were done using Brain Vision Analyzer (BrainProducts), MATLAB version 12.1 (The MathWorks), X code (Cohen, 2014), and Fieldtrip (Oostenveld et al., 2011) software.

To increase the signal-to-noise ratio and decrease the number of comparisons, we used the data from a pilot study $(n=19)$ to preselect our channels, frequencies, and time windows of interest for statistical testing (see Fig. $3 a, b$ ). The pilot study was identical to the main experiment with the exception that participants did not indicate the quality of their decision on each trial. We created six regions of interest (electrode selections): occipital, left and right parietal, and left and right motor and prefrontal, thereby focusing our analyses on changes in theta-band (4-6 $\mathrm{Hz})$ and lower beta-band $(13-20 \mathrm{~Hz})$ activity in an early $(0-1 \mathrm{~s})$ and late (2-2.5 s) time window after stimulus presentation (see Fig. $3 b$ ). We performed random-effects analyses by applying paired $t$ tests (two-tailed) to test whether the mean percentage of power change in each time window for each frequency bin differed significantly from baseline (from -0.3 to 0 s relative to stimulus onset). Because we tested six poolings in each time window and frequency bin, we corrected for multiple comparisons by adjusting the $p$-value by fixing the false discovery rate (FDR) at 0.05 (Benjamini and Hochberg, 1995).

Crucially, to study the relationship between theta- and beta-power modulations and trial-by-trial differences in metacognitive strategy, metacognitive adequacy, and task accuracy, a robust multiple regression was computed that estimated parameters for mean power in the abovedescribed time windows and frequency bands. This resulted in the following linear equation: 
a

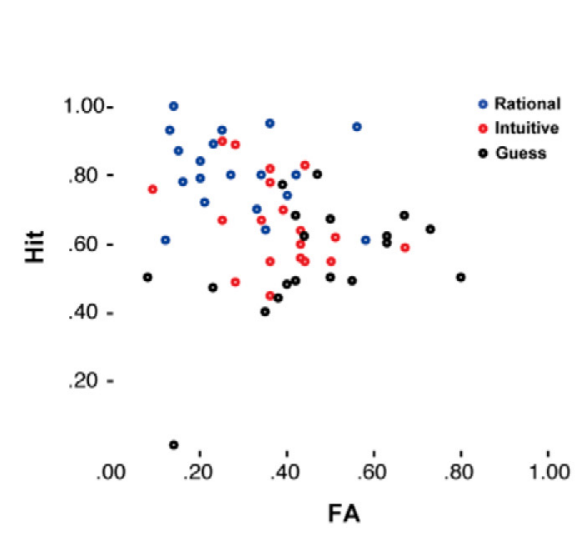

C

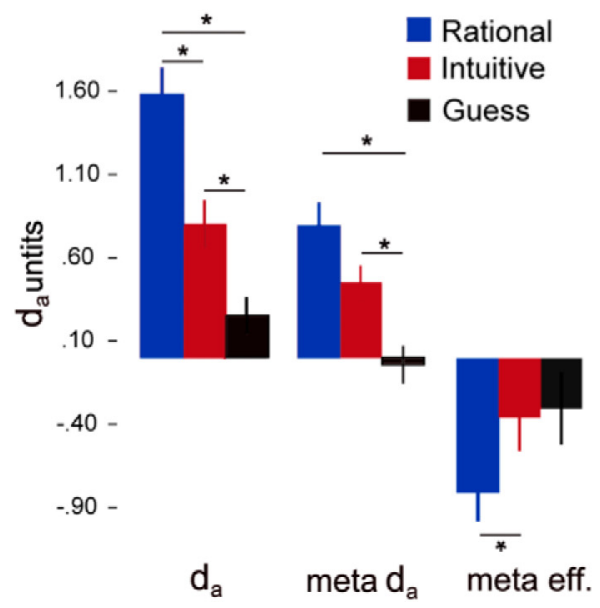

b

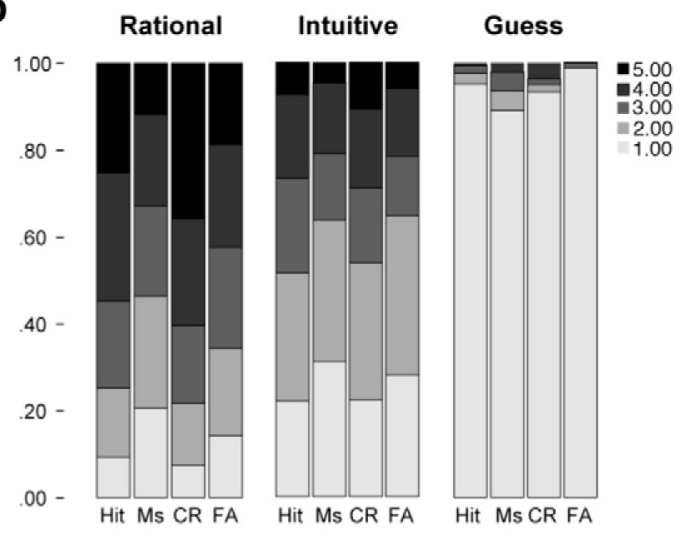

d

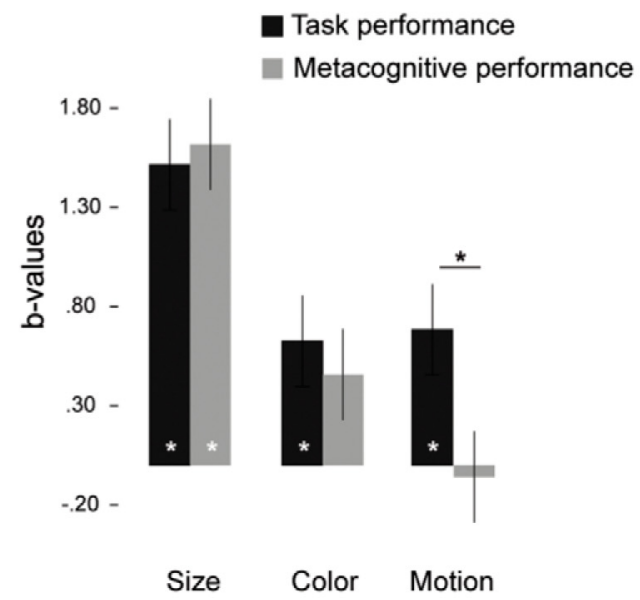

Figure 2. Behavioral results. $\boldsymbol{a}$, Hit and false alarm (FA) rates for rational, intuitive decisions, and guesses. $\boldsymbol{b}$, Proportions of all ratings given a hit, miss (MS), correct rejection (CR), and false alarm (FA). $\boldsymbol{c}$, For each decision strategy, $d_{a^{\prime}}$ meta- $d_{a^{\prime}}$ and metacognitive efficiency are displayed. $\boldsymbol{d}$, Regression coefficients demonstrate that different sources of information (size, color, and motion direction) contribute differentially to first-order task performance (black bars) and metacognitive performance (gray bars). Data are shown as means \pm (between-subject) SEM.

$\mathrm{Y}=\mathrm{INT}+\mathrm{b}_{1}$ StraJudg $+\mathrm{b}_{2}$ MetaAdeq $+\mathrm{b}_{3}$ TaskAcc $+\mathrm{b}_{4}$ ColorEv

$$
+\mathrm{b}_{5} \operatorname{SizeEv}+\mathrm{b}_{6} \text { MotionEv }+\mathrm{E}
$$

Where $Y$ is the data vector (baseline-corrected theta- or beta-power values across trials for each time period); INT is the intercept; $b_{1-6}$ are regression coefficients; $E$ is unexplained variance; and StratJudg, MetaAdeq, TaskAcc, ColorEv, SizeEv, and MotionEv are trial vectors of the participant's strategic judgment ratings, metacognitive judgment adequacy scores, first-order performance scores, and stimulus parameters on each trial. Strat)udg (metacognitive strategy judgment) ranged between 1 and 3 where 1 = guess, $2=$ intuitive decision, and $3=$ rational decision. We grouped trials again into 100 bins per participant. To be able to compare $b$ values across time, frequencies, poolings, and participants, we standardized them by scaling the regression coefficients by their SDs.

\section{Results}

Behavior

To test how strategic judgment related to task performance and metacognitive sensitivity, we performed a repeated-measures MANOVA on $d_{\mathrm{a}}$, meta $d_{\mathrm{a}}$, and metacognitive efficiency. We found a significant effect of strategic judgment for both $d_{\mathrm{a}}$ $\left(F_{(2,36)}=44.74 p<0.001\right)$ and meta- $d_{\mathrm{a}}\left(F_{(2,36)}=10.52 p<\right.$ $0.001)$ but observed a marginally significant/nonsignificant effect for metacognitive efficiency $\left(F_{(2,36)}=2.64 p=0.086\right)$. Participants were better able to distinguish sick from healthy patterns when making rational decisions compared with intuitive and guess trials (ratio-intuitive $d_{\mathrm{a}}: t_{(18)}=7.21, p<0.001$; ratio-guess $\left.d_{\mathrm{a}}: t_{(18)}=8.31, p<0.001\right)$, whereas performance on intuitive trials was better than guesses $\left(d_{\mathrm{a}}: t_{(18)}=3.65, p=0.002\right.$; Fig. $\left.2 a, c\right)$. We did not observe higher metacognitive sensitivity when participants made rational decisions compared with intuitive decisions (ratio-intuitive meta- $d_{\mathrm{a}}: t_{(18)}=1.87, p=0.078$ ). We did observe higher metacognitive sensitivity when participants made rational decisions compared with guesses (ratio-guess meta- $d_{\mathrm{a}}: t_{(18)}=$ $4.55, p<0.001)$ and intuitive decision compared with guesses (meta- $\left.\mathrm{d}_{\mathrm{a}}: t_{(18)}=2.71, p=0.014\right)$. When we compared metacognitive efficiency ( meta $d_{\mathrm{a}}-d_{\mathrm{a}}^{\prime}$ ), we observed higher efficiency on intuitive trials than on rational trials (metacognitive efficiency: $\left.t_{(18)}=2.73, p=0.014\right)$. The latter result demonstrates that the increase in $d^{\prime}$ is not proportionally reflected in the increase in meta $d^{\prime}$ when participants reported making use of a rational decision strategy. For the proportions of all ratings given a hit, correct rejection, false alarm, and miss, see Figure $2 b$.

To determine whether different stimulus parameters (size, color, and motion direction) contributed differentially to first-order task accuracy and metacognitive adequacy, we performed multiple linear regressions (Fig. 2d). Interestingly, we observed significant positive regression coefficients for the size, color, and motion direction indicators with respect to first-order task accuracy (all $t>3.18$, all 
a

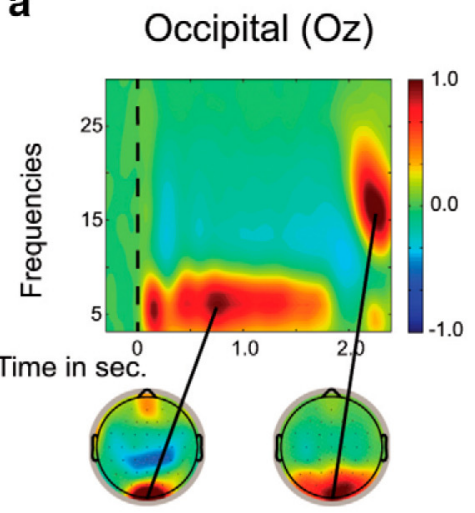

Anterior Frontal (AFz)

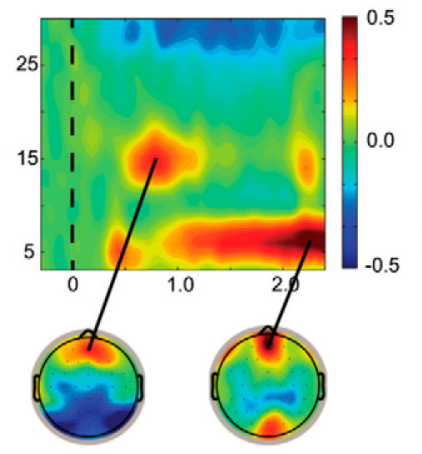

b

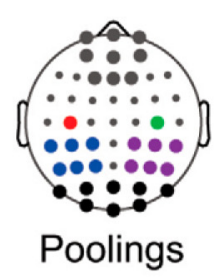

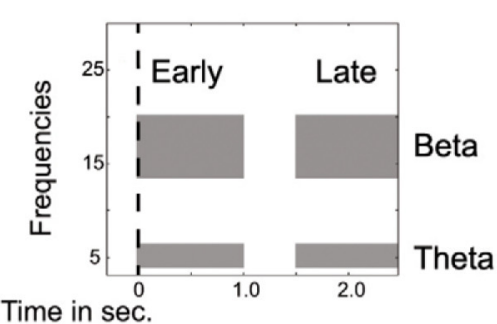

Time in sec.

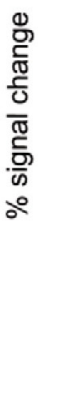

C
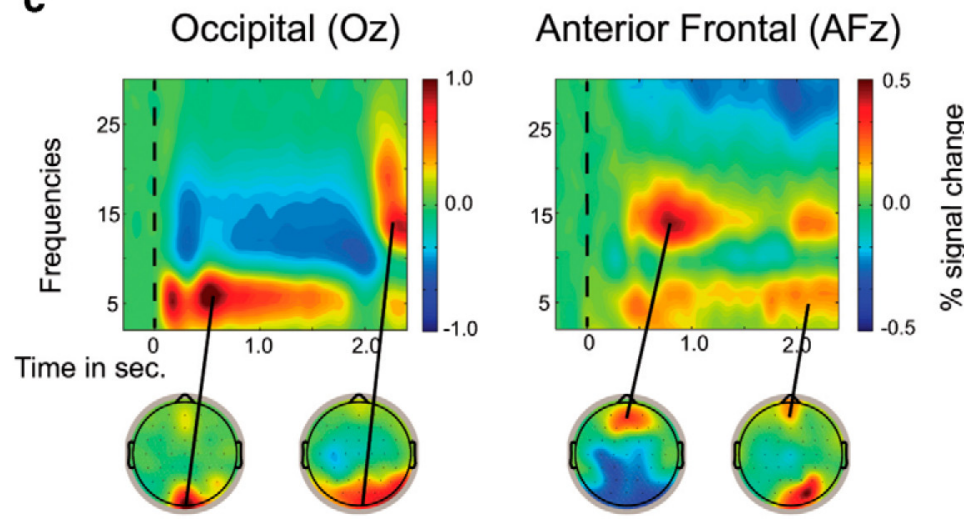

Figure 3. Time-frequency analyses. $\boldsymbol{a}$, We used the data from a pilot study $(n=19)$ to preselect our channels, frequencies, and time windows of interest for statistical testing. $\boldsymbol{b}$, Channels, frequencies, and time windows of interest. $\boldsymbol{c}$, In the first second after stimulus presentation, we observed increased theta power in the frontal and occipital channels. In this same time window, we found decreased beta-band activity in the left and right parietal, occipital, and left and right motor channels. In the late time window after stimulus presentation, we observed increased theta-band activity in frontal channels, whereas we found a decrease in theta-band activity in the left and right motor channels. In this later time window, we observed decreased beta-band activity in frontal, left-right parietal, and left-right motor channels.

$p<0.01$ ), but for metacognitive adequacy, we only observed significant positive regression coefficients with respect to the size indicator $\left(t_{(18)}=5.66, p<0.01\right)$. When we compared regression coefficients directly between first-order task accuracy and metacognitive adequacy, we observed that $b$ values for the motion indicator were significantly lower for metacognitive adequacy $\left(t_{(18)}=3.04, p<0.01\right)$. These findings are consistent with the answers participants provided when being asked explicitly at the end of the experiment about what kind of information they used for their decisions: Seventeen participants indicated that they had made use of size information, six that they had made use of color information, and six that they had made use of motion information.

Our behavioral findings indicate that presented sensory evidence differentially support first-order task performance and

second-order judgments (Charles et al., 2014; Maniscalco and Lau, 2016).

\section{EEG results}

We focused our analyses on two preselected time windows, six poolings and two frequency bands derived from data from a pilot study (Fig. $3 a, b$ ). In the first second after stimulus presentation we found increased theta power in frontal $\left(t_{(18)}=\right.$ 3.66, $p<0.05$, FDR-corrected) and occipital $\left(t_{(18)}=5.20, p<0.05\right.$, FDRcorrected) channels compared with baseline. In this same time window, we found decreased beta-band activity in left and right parietal, occipital, and left and right motor channels (all $t_{(18)}>3.59, p<0.05$, FDR-corrected). In the late time window after stimulus presentation (1.5-2.5 s), we observed increased theta-band activity in frontal channels $\left(t_{(18)}=2.90, p<0.05\right.$, FDR-corrected), whereas we found a decrease in theta-band activity in left and right motor channels $t_{(18)}=3.46$ and $t_{(18)}=2.88$, respectively, $p<0.05$, FDRcorrected). In this later time window, we observed decreased beta-band activity in frontal, left-right parietal, and left-right motor channels (all $t_{(18)}>2.48, p<0.05$, FDR-corrected; Fig. 3c).

In the present study, we were specifically interested in how variations in first-order task accuracy, metacognitive adequacy, and metacognitive strategy judgment are related to changes in oscillation power. We therefore performed a multiple linear regression (Cohen, 2014) to study the relationship between theta- and beta-power changes and diagnosis accuracy, metacognitive adequacy, and strategy judgment while partialing out shared explained variance among the variables entered into the regression (i.e., stimulus properties, task accuracy, metacognitive adequacy, and strategy judgment; see Materials and Methods). Figures 4 and 5 show the multiple regression coefficients for the regression terms task accuracy, metacognitive adequacy, and strategy judgment. We tested whether regression coefficients differed significantly from zero for each frequency band and time window separately $(p<0.05$, FDR-corrected; significant poolings are indicated with asterisks). We observed a positive linear relationship between early $\left(t_{(18)}=3.16, p<0.05\right.$, FDR-corrected) and late $\left(t_{(18)}=3.64, p<0.05\right.$, FDR-corrected) prefrontal thetaband activity and metacognitive adequacy, whereas we found a negative linear relationship between late right motor beta-band activity and first-order task accuracy $\left(t_{(18)}=3.07, p<0.05\right.$, FDR-corrected). These results demonstrate that theta-band activity in prefrontal channels relates selectively to metacognitive adequacy. Variance in metacognitive strategy judgment was not associated significantly with oscillation power in either the early or late theta- or beta-bands. To test whether coefficients actually differed significantly between task accuracy and metacognitive adequacy (cf. Nieuwenhuis et al., 


\section{a Theta Power}

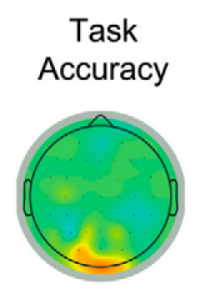

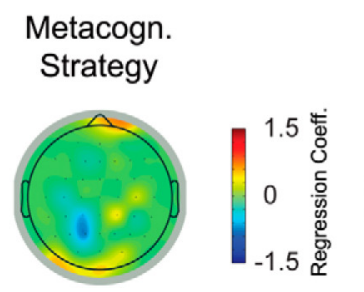

b Beta Power

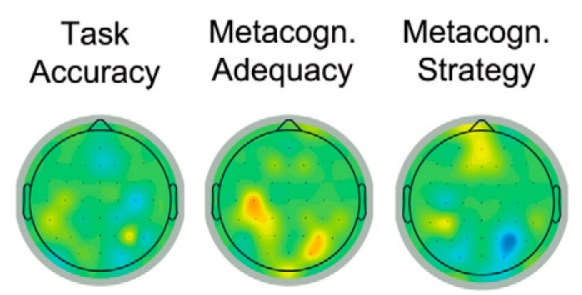

Figure 4. Multiple linear regression EEG results: early time window ( $0-1 \mathrm{~s})$. We performed a multiple linear regression to study the relationship between theta- and beta-power changes and diagnosis accuracy, metacognitive adequacy, and strategy judgment while partialing out shared explained variance among the variables. $\boldsymbol{a}$, We observed a positive linear relationship between prefrontal theta-band activity and metacognitive adequacy. $\boldsymbol{b}$, We found no effects for beta power. Asterisks indicate significant poolings.

\section{a Theta Power}

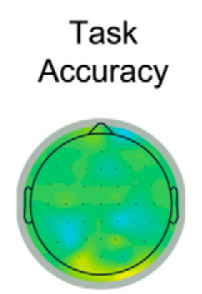

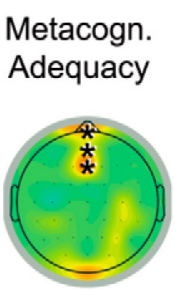

b Beta Power
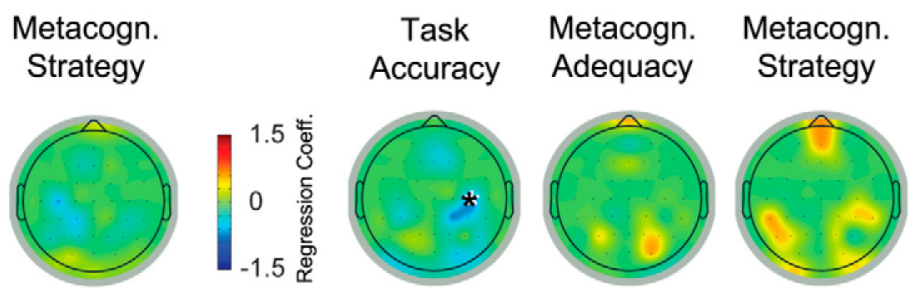

Figure 5. Multiple linear regression EEG results: late time window (1.5-2.5 s). $\boldsymbol{a}$, We also observed a positive linear relationship between prefrontal theta-band activity and metacognitive adequacy in the late time window. $\boldsymbol{b}$, In this time window, we also observed a negative linear relationship between late right motor beta-band activity and first-order task accuracy. Asterisks indicate significant poolings.

2011), we compared those coefficients directly for prefrontal theta and found that regression coefficients were higher for metacognitive adequacy compared with first-order task accuracy in the early $\left(t_{(18)}=2.56, p=0.02\right)$ and late $\left(t_{(18)}=2.69, p=0.03\right)$ time windows.

In the present study, we observed a relationship between theta and metacognitive performance in a time window before the first-order response (i.e., the first $2.5 \mathrm{~s}$ after stimulus onset). We constructed the task in such a way that we expected participants to have reached a first- and second-order decision before giving a first-order response. For that purpose (and for the purpose of prolonged evidence accumulation; see above), we added a jittered 1-1.5 s time window between stimulus offset and the onset of instructions to respond. To further investigate the relationship between metacognitive performance and theta-band activity, it would be interesting to examine the time window right before the second-order response. Unfortunately, we did not add a time window between first- and second-order responses. Nonetheless, we performed an additional analysis time locking the epochs to the second-order response (using $1 \mathrm{~s}$ of data before the first-order response). We did not observe a significant effect $\left(t_{(18)}=1.40\right.$, $p>0.05$, FDR-corrected). However, this result should be interpreted with great caution. Indeed, because of the above described relatively long and jittered time window between stimulus offset and first-order response, the timing of stimulus onset varied per epoch when time locking epochs to the second-order response. In dominant models of metacognition, stimulus onset is taken as the starting point of first- and second-order decisions. In future studies, it would be very interesting to investigate the relationship between theta and metacognitive performance in a distinct time window directly preceding second-order responses.

\section{Discussion}

To summarize, we applied multiple linear regression analyses to our behavioral and electrophysiological data to determine the relationship between first- and second-order performance. Results demonstrate that sources of sensory evidence contributing to first-order decision making do not similarly support secondorder decision making. Variance in first-order diagnosis performance was driven by size, color, and motion information, whereas variance in metacognitive adequacy was driven exclusively by size information. These findings suggest that part of the sensory evidence used for first-order performance becomes inaccessible or becomes susceptible to decay and noise when arriving at the stage at which this sensory information is being used for metacognitive judgments (Pleskac and Busemeyer, 2010; Charles et al., 2014; Maniscalco and Lau, 2016).

To determine whether we could distinguish oscillatory mechanisms specifically related to first- and second-order task performance, we applied multiple linear regression analyses to our EEG data. We observed a positive relation between prefrontal thetaband activity and metacognitive performance that could not be explained by first-order task performance or the various stimulus parameters. Further, we found that increased task accuracy related to decreased beta power in motor regions (see also Donner et al., 2007, 2009).

In a recent study, Maniscalco and Lau (2016) compared three dominant models that describe the relationship between objective task performance and metacognitive (subjective) task performance. In their study, they compared single-channel models, which presume that the same sources of (and quality of) information support both first- and second-order task performance; 
dual channel models, which presume that two processing streams differentially give rise to first- and second-order task performance; and hierarchical models, which presume that a lateprocessing stage evaluates the quality of sensory processing. The results from comparing these models demonstrated that dissociations between first- and second-order performance are best captured by hierarchical models. Maniscalco and Lau (2016) concluded that hierarchical models performed best due to the fact that such models require a less stringent relationship between the quality of information available for first- and second-order task performance.

These results are consistent with simulations demonstrating that a second-order network is able to learn gradually to interpret contingencies related to processing in first-order neural networks (Cleeremans et al., 2007). From this point of view, second-order networks could learn to evaluate the extent to which activity patterns in brain regions contributing to first-order decision making result in successful performance.

Here, we observed that part of the information strongly supporting first-order task performance (size information; Fig. $2 d$ ) also supported metacognitive performance, whereas "weaker" information (color and motion; Fig. $2 d$ ) contributed exclusively to first-order task performance. These results indicate that the quality of information used for first-order performance is not similar to the quality of information used for second-order performance, possibly due to accrual of noise or signal decay (Pleskac and Busemeyer, 2010; Maniscalco and Lau, 2016). Alternatively, different sources of information can be accessible differentially for first- and second-order processes (Del Cul et al., 2009). From this perspective, size information can be seen as information processed in the "conscious" channel, whereas color and motion information are being processed in the "unconscious" channel. Although such dual-channel models did not seem to capture most accurately dissociations between first- and second-order task performance in a visual backward-masking task (Maniscalco and Lau, 2016), it remains to be tested whether this generalizes to other tasks or the dissociations between firstand second-order task performance that we observed in the present study (Fig. 2).

\section{Information used for first- and second-order task performance}

Recent work suggest that sensory evidence supporting first-order performance can become supplemented by additional sources of information that become available after a first-order decision has been made (Wierzchoń et al., 2014; Fleming et al., 2015). For instance, it has been demonstrated that the manipulation of neural activity in premotor cortex affects metacognitive performance without altering first-order accuracy (Fleming et al., 2015). Further, when participants had to rate the visibility of a stimulus before making a first-order decision, exhaustiveness of the scales was lower (though not for confidence judgments) compared with the situation in which participants provided such ratings after responding to the stimulus (Wierzchon et al., 2014). The investigators concluded that the identification task decisions affected the subsequent awareness ratings. These findings suggest that metacognition might be an "embodied" process in which sensory evidence becomes integrated with motor and body-related information (Fleming et al., 2015; Allen et al., 2016). The availability of postdecision information that is only accessible for metacognitive processes could also contribute to the observed different relationship between presented sensory evidence and first- and second-order task performance (Fig. $2 d$ ).
However, in the present study, we did not observe a relationship between activity in motor channels and metacognitive adequacy, whereas we did observe a link between sensorimotor beta-band activity and first-order task performance. In our analyses, we focused on the time period before first-order responses, suggesting that motor preparatory processes at least do not contribute to metacognitive performance. It could be that the actual motor execution (and a possible accompanying "sense of fluency") is necessary to contribute to metacognitive performance. At the present, however, this still remains to be investigated.

Here, we did observe a relationship between first-order responses and beta-band activity in motor regions, as reported previously by Donner et al. (2009). It is currently hotly debated in what way the action system is involved in decision making (Cisek and Kalaska, 2005; Rushworth et al., 2012; Calderon et al., 2016); specifically, whether action selection depends on a serial or a parallel cognitive architecture. Our present findings could be interpreted as the result of continuous interactions between perceptual and action systems, which are more effective in the case of correct decisions. Alternatively, the observed effect may reflect a late processing stage, and predictive activity of decision outcome might have also been evident in other regions when using more spatially refined imaging methods or recordings at the singleneuron level (Donner et al., 2009). Nonetheless, our findings demonstrate that activity related to motor preparation can be predictive of task accuracy.

\section{Prefrontal theta oscillations}

The present study indicates that metacognitive processes are orchestrated by means of prefrontal theta oscillations (Figs. 4, 5). Consistent with our findings, previous work demonstrated that lesions to prefrontal cortex induce metacognitive deficits without necessarily disrupting first-order performance (Pannu and Kaszniak, 2005; Fleming et al., 2014). Similarly, modulating prefrontal cortical activity via theta burst stimulation has been shown to alter metacognitive performance without affecting first-order decision making (Rounis et al., 2010; Ryals et al., 2016). In the present study, we observed that theta power specifically in prefrontal channels was related to metacognitive performance (Figs. 4, 5).

A large body of work indicates that flexible and adaptive behavior and prefrontal theta oscillations are intimately related. It has been shown that prefrontal theta oscillations support implementation of cognitive control, action monitoring, and flexible behavior (Cavanagh and Frank, 2014; Cohen, 2014). Theta-band mechanisms are thought to facilitate flexible connections between prefrontal cortex and lower-tier task-related networks and allow for top-down modulation to adjust ongoing behavior ( $\mathrm{Co}$ hen et al., 2009; Cohen and Cavanagh, 2011; Van Driel et al., 2015). In clinical populations, dysfunction of prefrontal thetaphase dynamics has been linked recently to adaptive behavior deficits in schizophrenia (Reinhart et al., 2015). Using direct current stimulation over frontal cortex, Reinhart et al. (2015) demonstrated that adaptive control (post-error slowing) in schizophrenia patients increased after frontal electrical stimulation. This behavioral effect coincided with a change in the organization of theta-band phase dynamics. Interestingly, previous work associated schizophrenia with metacognitive deficits (Moritz and Woodward, 2002, 2007). Individuals with schizophrenia demonstrated impaired discriminatory capabilities between correct and incorrect judgments, as reflected in confidence ratings (Moritz et al., 2006). Here, the nature of the observed relationship between theta power in prefrontal channels and 
metacognitive performance remains an open question. We observed the effect well before the second-order response had been made, opening the possibility that the link between metacognitive performance and prefrontal theta could be due to more general processes that support metacognition performance. Further, the way that typical measures of cognitive control (e.g., posterror slowing, response conflict/inhibition; Rabbitt, 1966; Ridderinkhof et al., 2004; Charles et al., 2013; Wokke et al., 2016) and metacognition are related (Boldt and Yeung, 2015) remains an interesting open empirical question. From this perspective, metacognition could be seen as the internalization of an initially external process making use of similar neural mechanisms and enabling us to guide behavior more effectively (Buzsáki et al., 2014).

\section{References}

Allen M, Fardo F, Dietz MJ, Hillebrandt H, Friston KJ, Rees G, Roepstorff A (2016) Anterior insula coordinates hierarchical processing of tactile mismatch responses. Neuroimage 127:34-43. CrossRef Medline

Baranski JV, Petrusic WM (1998) Probing the locus of confidence judgments: experiments on the time to determine confidence. J Exp Psychol Hum Percept Perform 24:929-945. CrossRef Medline

Benjamini Y, and Hochberg Y (1995) Controlling the false discovery rate: a practical and powerful approach to multiple testing. Journal of the Royal Statistical Society Series B 85:289-300.

Berry D, Dienes ZP (1993) Implicit learning: theoretical and empirical issues. Erlbaum: Psychology Press.

Boldt A, Yeung N (2015) Shared neural markers of decision confidence and error detection. J Neurosci 35:3478-3484. CrossRef Medline

Buzsáki G, Peyrache A, Kubie J (2014) Emergence of cognition from action. Cold Spring Harb Symp Quant Biol 79:41-50. CrossRef Medline

Calderon CB, Verguts T, Gevers W (2015) Losing the boundary: Cognition biases action well after action selection. Journal of Experimental Psychology: General 144:737. CrossRef

Cavanagh JF, Frank MJ (2014) Frontal theta as a mechanism for cognitive control. Trends Cogn Sci 18:414-421. CrossRef Medline

Charles L, Van Opstal F, Marti S, Dehaene S (2013) Distinct brain mechanisms for conscious versus subliminal error detection. Neuroimage 73: 80-94. CrossRef Medline

Charles L, King JR, Dehaene S (2014) Decoding the dynamics of action, intention, and error detection for conscious and subliminal stimuli. J Neurosci 34:1158-1170. CrossRef Medline

Cisek P, Kalaska JF (2005) Neural correlates of reaching decisions in dorsal premotor cortex: specification of multiple direction choices and final selection of action. Neuron 45:801-814. CrossRef Medline

Cleeremans A, Destrebecqz A, Boyer M (1998) Implicit learning: news from the front. Trends Cogn Sci 2:406-416. CrossRef Medline

Cleeremans A, Timmermans B, Pasquali A (2007) Consciousness and metarepresentation: a computational sketch. Neural Netw 20:1032-1039. CrossRef Medline

Cohen MX (2014) Analyzing neural time series data: theory and practice. Cambridge, MA: MIT.

Cohen MX, Cavanagh JF (2011) Single-trial regression elucidates the role of prefrontal theta oscillations in response conflict. Front Psychol 2:30. CrossRef Medline

Cohen MX, van Gaal S, Ridderinkhof KR, Lamme VA (2009) Unconscious errors enhance prefrontal-occipital oscillatory synchrony. Front Hum Neurosci 3:54. CrossRef Medline

Del Cul A, Dehaene S, Reyes P, Bravo E, Slachevsky A (2009) Causal role of prefrontal cortex in the threshold for access to consciousness. Brain 132: 2531-2540. CrossRef Medline

Desender K, Van Opstal F, Hughes G, Van den Bussche E (2016) The temporal dynamics of metacognition: Dissociating task-related activity from later metacognitive processes. Neuropsychologia 82:54-64. CrossRef Medline

Donner TH, Siegel M, Oostenveld R, Fries P, Bauer M, Engel AK (2007) Population activity in the human dorsal pathway predicts the accuracy of visual motion detection. J Neurophysiol 98:345-359. CrossRef Medline

Donner TH, Siegel M, Fries P, Engel AK (2009) Buildup of choice- predictive activity in human motor cortex during perceptual decision making. Curr Biol 19:1581-1585. CrossRef Medline

Donner TH, Siegel M (2011) A framework for local cortical oscillation patterns. Trends Cogn Sci 15:191-199. CrossRef Medline

Engel AK, Fries P (2010) Beta-band oscillations-signalling the status quo? Curr Opin Neurobiol 20:156-165. CrossRef Medline

Flavell JH (1979) Metacognition and cognitive monitoring: A new area of cognitive-developmental inquiry. American Psychologist 34:906. CrossRef

Fleming SM, Dolan RJ (2012) The neural basis of metacognitive ability. Philos Trans R Soc Lond B Biol Sci 367:1338-1349. CrossRef Medline

Fleming SM, Lau HC (2014) How to measure metacognition. Front Hum Neurosci 8:443. CrossRef Medline

Fleming SM, Ryu J, Golfinos JG, Blackmon KE (2014) Domain-specific impairment in metacognitive accuracy following anterior prefrontal lesions. Brain 137:2811-2822. CrossRef Medline

Fleming SM, Maniscalco B, Ko Y, Amendi N, Ro T, Lau H (2015) Actionspecific disruption of perceptual confidence. Psychol Sci 26:89-98. CrossRef Medline

Haegens S, Nácher V, Hernández A, Luna R, Jensen O, Romo R (2011) Beta oscillations in the monkey sensorimotor network reflect somatosensory decision making. Proc Natl Acad Sci U S A 108:10708-10713. CrossRef Medline

Harsay HA, Spaan M, Wijnen JG, Ridderinkhof KR (2012) Error awareness and salience processing in the oddball task: shared neural mechanisms. Front Hum Neurosci 6:246. CrossRef Medline

Hebart MN, Schriever Y, Donner TH, Haynes JD (2016) The relationship between perceptual decision variables and confidence in the human brain. Cereb Cortex 26:118-130. CrossRef Medline

Kalcher J, Pfurtscheller G (1995) Discrimination between phase-locked and non-phase-locked event-related EEG activity. Electroencephalography and clinical neurophysiology 94:381-384. CrossRef Medline

Kiani R, Shadlen MN (2009) Representation of confidence associated with a decision by neurons in the parietal cortex. science 324:759-764. CrossRef Medline

Kiani R, Corthell L, Shadlen MN (2014) Choice certainty is informed by both evidence and decision time. Neuron 84:1329-1342. CrossRef Medline

King JR, Dehaene S (2014) A model of subjective report and objective discrimination as categorical decisions in a vast representational space. Philos Trans R Soc Lond B Biol Sci 369:20130204. CrossRef Medline

Kloosterman NA, Meindertsma T, Hillebrand A, van Dijk BW, Lamme VA, Donner TH (2015) Top-down modulation in human visual cortex predicts the stability of a perceptual illusion. J Neurophysiol 113:1063-1076. CrossRef Medline

Macmillan NA, Creelman CD (2004) Detection theory: a user's guide. Cambridge: Psychology Press.

Maniscalco B, Lau H (2012) A signal detection theoretic approach for estimating metacognitive sensitivity from confidence ratings. Conscious Cogn 21:422-430. CrossRef Medline

Maniscalco B, Lau H (2016) The signal processing architecture underlying subjective reports of sensory awareness. Neurosci Conscious 2016: pii: niw002. CrossRef Medline

Mitra PP, Pesaran B (1999) Analysis of dynamic brain imaging data. Biophys J 76:691-708. CrossRef Medline

Moritz S, Woodward TS (2002) Memory confidence and false memories in schizophrenia. J Nerv Ment Dis 190:641-643. CrossRef Medline

Moritz S, Woodward TS, Rodriguez-Raecke R (2006) Patients with schizophrenia do not produce more false memories than controls but are more confident in them. Psychol Med 36:659-667. CrossRef Medline

Moritz S, Woodward TS (2007) Metacognitive training in schizophrenia: from basic research to knowledge translation and intervention. Curr Opin Psychiatry 20:619-625. CrossRef Medline

Nieuwenhuis S, Forstmann BU, Wagenmakers EJ (2011) Erroneous analyses of interactions in neuroscience: a problem of significance. Nat Neurosci 14:1105-1107. CrossRef Medline

Oostenveld R, Fries P, Maris E, Schoffelen JM (2011) FieldTrip: open source software for advanced analysis of MEG, EEG, and invasive electrophysiological data. Comput Intell Neurosci 2011:156869. CrossRef Medline

Pannu JK, Kaszniak AW (2005) Metamemory experiments in neurological populations: a review. Neuropsychol Rev 15:105-130. CrossRef Medline Pleskac TJ, Busemeyer JR (2010) Two-stage dynamic signal detection: a the- 
ory of choice, decision time, and confidence. Psychol Rev 117:864-901. CrossRef Medline

Price MC, Norman E (2008) Intuitive decisions on the fringes of consciousness: are they conscious and does it matter? Judgment and Decision Making 3:28.

Rabbitt PM (1966) Errors and error correction in choice-response tasks. J Exp Psychol 71:264-272. CrossRef Medline

Reinhart RM, Zhu J, Park S, Woodman GF (2015) Medial-frontal stimulation enhances learning in schizophrenia by restoring prediction error signaling. J Neurosci 35:12232-12240. CrossRef Medline

Ridderinkhof KR, Ullsperger M, Crone EA, Nieuwenhuis S (2004) The role of the medial frontal cortex in cognitive control. Science 306:443-447. CrossRef Medline

Rounis E, Maniscalco B, Rothwell JC, Passingham RE, Lau H (2010) Thetaburst transcranial magnetic stimulation to the prefrontal cortex impairs metacognitive visual awareness. Cogn Neurosci 1:165-175. CrossRef Medline

Rousselet GA, Husk JS, Pernet CR, Gaspar CM, Bennett PJ, Sekuler AB (2009) Age-related delay in information accrual for faces: evidence from a parametric, single-trial EEG approach. BMC Neurosci 10:114. CrossRef Medline

Rushworth MF, Kolling N, Sallet J, Mars RB (2012) Valuation and decision making in frontal cortex: one or many serial or parallel systems? Curr Opin Neurobiol 22:946-955. CrossRef Medline

Ryals AJ, Rogers LM, Gross EZ, Polnaszek KL, Voss JL (2016) Associative recognition memory awareness improved by theta-burst stimulation of frontopolar cortex. Cereb Cortex 26:1200-1210. CrossRef Medline

Siegel M, Donner TH, Engel AK (2012) Spectral fingerprints of large-scale neuronal interactions. Nat Rev Neurosci 13:121-134. CrossRef Medline
Steinhauser M, Yeung N (2010) Decision processes in human performance monitoring. J neuroscience 30:15643-15653. CrossRef Medline

van Driel J, Swart JC, Egner T, Ridderinkhof KR, Cohen MX (2015) (No) time for control: frontal theta dynamics reveal the cost of temporally guided conflict anticipation. Cogn Affect Behav Neurosci 15:787-807. CrossRef Medline

Van Vugt MK, Simen P, Nystrom LE, Holmes P, and Cohen JD (2012) EEG oscillations reveal neural correlates of evidence accumulation. Front Neurosci 6:106. CrossRef

Vigário RN (1997) Extraction of ocular artefacts from EEG using independent component analysis. Electroencephalogr Clin Neurophysiol 103: 395-404. CrossRef Medline

Weiskrantz L, Warrington EK, Sanders MD, Marshall J (1974) Visual capacity in the hemianopic field following a restricted occipital ablation. Brain 97:709-728. CrossRef Medline

Werkle-Bergner M, Grandy TH, Chicherio C, Schmiedek F, Lövdén M, Lindenberger U (2014) Coordinated within-trial dynamics of lowfrequency neural rhythms controls evidence accumulation. J Neurosci 34:8519-8528. CrossRef Medline

Wierzchoń M, Paulewicz B, Asanowicz D, Timmermans B, Cleeremans A (2014) Different subjective awareness measures demonstrate the influence of visual identification on perceptual awareness ratings. Conscious Cogn 27:109-120. CrossRef Medline

Wokke ME, Knot SL, Fouad A, Richard Ridderinkhof K (2016) Conflict in the kitchen: Contextual modulation of responsiveness to affordances. Conscious Cogn 40:141-146. CrossRef Medline

Yeung N, Summerfield C (2012) Metacognition in human decision making: confidence and error monitoring. Philos Trans R Soc Lond B Biol Sci 367:1310-1321. CrossRef Medline 